01

\title{
Канонические алгоритмы численного интегрирования уравнений движения заряженных частиц
}

\author{
(С) И.Н. Ефримов, Е.А. Морозов, А.Р. Морозова
}

Ижевский государственный технический университет им. М.Т. Калашникова, 427622 Ижевск, Россия

e-mail: chti@chti.ru

(Поступило в Редакцию 13 июля 2015 г. В окончательной редакции 29 марта 2016 г.)

Рассмотрен метод численного интегрирования уравнений движения заряженных частиц в магнитном поле, в основе которого лежат канонические преобразования фазового пространства гамильтоновой механики. Использование канонических преобразований обеспечивает устойчивость процесса интегрирования к накоплению погрешности счета. Алгоритмы интегрирования содержат минимально возможное количество арифметических операций и могут быть использованы для расчета ускорителей и приборов электронной и ионной оптики.

DOI: 10.21883/JTF.2017.02.44121.1519

\section{Введение}

В основе гамильтоновой механики лежат канонические преобразования фазового пространства координат и импульсов, сохраняющие элемент его объема [1]. В частности, механическое движение рассматривается как частный случай такого преобразования. В работе [2] рассматриваются алгоритмы численного интегрирования уравнений Гамильтона, представляющие собой бесконечно малые канонические преобразования. В этом случае следствием сохранения элемента объема является устойчивость алгоритмов к накоплению погрешности счета, а сам процесс численного интегрирования воспроизводит движение исходной системы в условиях малого консервативного возмущения. Отсутствие накопления погрешности счета в каноническом методе позволяет исследовать эволюцию системы на протяжении больших интервалов времени, что невозможно при использовании традиционных методов Эйлера и Рунге-Кутта.

Указанные свойства канонического метода делают перспективным его использование для численного интегрирования движения заряженных частиц в ускорителях и приборах электронной и ионной оптики. Поставим задачу найти алгоритмы интегрирования уравнений движения заряженных частиц в магнитном поле, которые осуществляют каноническое преобразование фазового пространства.

\section{Основные положения}

Рассмотрим движение частицы с массой $m$ и зарядом $e$ в магнитном поле [3]. В гамильтоновой механике магнитное поле характеризуется векторным потенциалом $\mathbf{A}$, который, в общем случае, является функцией радиусвектора частицы $\mathbf{r}$ и времени $t: \mathbf{A}=\mathbf{A}(\mathbf{r}, t)$. Функция Гамильтона, определяющая характер движения частицы в релятивистском случае, имеет вид

$$
H=\left[m^{2} c^{4}+c^{2}(\mathbf{P}-e \mathbf{A})^{2}\right]^{0.5},
$$

где $c$ - скорость света, $\mathbf{P}-$ обобщенный импульс частицы.

В нерелятивистском случае функции Гамильтона (1) принимает вид

$$
H=\frac{1}{2 m}(\mathbf{P}-e \mathbf{A})^{2} .
$$

Обобщенный импульс частицы связан с ее импульсом р выражением

$$
\mathbf{P}=\mathbf{p}+e \mathbf{A} .
$$

Уравнения Гамильтона, определяющие закон движения, имеют вид

$$
\dot{\mathbf{P}}=-\frac{\partial H}{\partial \mathbf{r}}, \quad \dot{\mathbf{r}}=\frac{\partial H}{\partial \mathbf{P}}
$$

Для сокращения записи будем использовать оператор набла $\nabla$, отмечая нижним индексом переменную, по которой берется производная, в частности, $\partial H / \partial \mathbf{P}=\nabla_{\mathbf{p}} H$, $\partial H / \partial \mathbf{r}=\nabla \mathbf{r} H$.

Линейное пространство $R^{6}=(\mathbf{r}, \mathbf{p})=\left(x_{1}, x_{2}, x_{3}, p_{1}\right.$, $\left.p_{2}, p_{3}\right)$ является фазовым пространством частицы. Преобразование фазового пространства

$$
\begin{aligned}
& \left(x_{1}^{(i)}, x_{2}^{(i)}, x_{3}^{(i)}, p_{1}^{(i)}, p_{2}^{(i)}, p_{3}^{(i)}\right) \\
& \rightarrow\left(x_{1}^{(i+1)}, x_{2}^{(i+1)}, x_{3}^{(i+1)}, p_{1}^{(i+1)}, p_{2}^{(i+1)}, p_{3}^{(i+1)}\right)
\end{aligned}
$$

называется каноническим, если для него выполнены условия [1]

$$
\begin{gathered}
{\left[p_{j}^{(i+1)}, x_{j}^{(i+1)}\right]=\frac{\partial p_{j}^{(i+1)}}{\partial p_{j}^{(i)}} \frac{x_{j}^{(i+1)}}{\partial x_{j}^{(i)}}-\frac{\partial p_{j}^{(i+1)}}{\partial x_{j}^{(i)}} \frac{x_{j}^{(i+1)}}{\partial p_{j}^{(i)}}=1,} \\
j=1,2,3, \quad i=1,2, \ldots
\end{gathered}
$$

Выражение (6) называется скобкой Пуассона. 
Для канонических преобразований справедлива теорема Лиувилля, они сохраняют фазовый объем, занимаемый системой частиц [1].

Зависящее от параметра $\tau$ преобразование (5) называется бесконечно малым параметрическим преобразованием, если выполнено условие

$$
\begin{gathered}
\tau \rightarrow 0, \quad\left(x_{1}^{(i+1)}, x_{2}^{(i+1)}, x_{3}^{(i+1)}, p_{1}^{(i+1)}, p_{2}^{(i+1)}, p_{3}^{(i+1)}, \tau\right) \\
\rightarrow\left(x_{1}^{(i)}, x_{2}^{(i)}, x_{3}^{(i)}, p_{1}^{(i)}, p_{2}^{(i)}, p_{3}^{(i)}, \tau\right) .
\end{gathered}
$$

Алгоритмы численного интегрирования уравнений Гамильтона (4) могут быть построены на основе использования преобразований (7), если параметр $\tau$ принять за шаг интегрирования.

Поставим задачу найти бесконечно малые параметрические преобразования фазового пространства (7) при выполнении условия каноничности (6) для различных случаев движения заряженных частиц в ускорителях и приборах электронной и ионной оптики.

\section{Нерелятивистская частица в постоянном магнитном поле}

В большинстве устройств, использующих магнитное поле для формирования и фокусировки потоков заряженных частиц, векторный потенциал обладает осевой симметрией, поэтому для исследования движения частиц в таких полях используют цилиндрическую систему координат. Пусть $O \rho \varphi z$ - такая система с осью $O z$, совпадающей с осью симметрии поля. В выбранной системе координат векторный потенциал имеет составляющие

$$
A_{\varphi}=A_{\varphi}(\rho, z), \quad A_{\rho}=A_{z}=0 .
$$

Пусть векторный потенциал (8) постоянен во времени, электрическое поле отсутствует, а скорость частицы много меньше скорости света. Такой случай, в частности, имеет место в электронных магнитных спектрометрах [4]. Запишем функцию Гамильтона (2) и динамические уравнения (4) в системе координат $O \rho \varphi z$, принимая без ограничения общности массу частицы, равной единице

$$
\begin{gathered}
H=0.5\left(P_{\rho}^{2}+P_{z}^{2}+\rho^{-2}\left(P_{\varphi}-e \rho^{2} A_{\varphi}\right)^{2}\right), \\
\dot{P}_{\rho}=-\rho\left(P_{\varphi}-e A_{\varphi}\right)\left(P_{\varphi}-e A_{\varphi}-e \rho \nabla_{\rho} A_{\varphi}\right), \\
\dot{\rho}=P_{\rho}, \quad \dot{P}_{\varphi}=0, \\
\dot{\varphi}=\left(P_{\varphi}-e A_{\varphi}\right), \quad \dot{P}_{z}=e \rho^{2}\left(P_{\varphi}-e A_{\varphi}\right) \nabla_{z} A_{\varphi}, \quad \dot{z}=P_{\varphi},
\end{gathered}
$$

где $P_{\rho}, P_{\varphi}, P_{z}$ - цилиндрические составляющие обобщенного импульса, $A_{\varphi}=A_{\varphi}(\rho, z)$. Из равенства нулю производной углового импульса следует его сохранение $P_{\varphi}=P_{0}=$ const. Введем следующие обозначения для правых частей (10):

$$
\begin{aligned}
& f_{\rho}=-\rho\left(P_{\varphi}-e A_{\varphi}\right)\left(P_{\varphi}-e A_{\varphi}-e \rho \nabla_{\rho} A_{\varphi}\right), \\
& f_{\varphi}=\left(P_{\varphi}-e A_{\varphi}\right), \quad f_{z}=e \rho^{2}\left(P_{\varphi}-e A_{\varphi}\right) \nabla_{z} A_{\varphi},
\end{aligned}
$$

и перепишем систему (10) в виде

$$
P_{\varphi}=P_{0}, \quad \dot{P}_{\rho}=f_{\rho}, \quad \dot{P}_{z}=f_{z}, \quad \dot{\varphi}=f_{\varphi}, \quad \dot{\rho}=P, \quad \dot{z}=P .
$$

Рассмотрим бесконечно малое по параметру $\tau$ преобразование фазового пространства, которое будет определять алгоритм численного интегрирования уравнений (10)

$$
\begin{gathered}
P_{\varphi}^{(i+1)}=P_{0}, P_{\rho}^{(i+1)}=P_{\rho}^{(i)}+f_{\rho} \tau, P_{z}^{(i+1)}=P_{z}^{(i)}+f_{z} \tau, \\
\varphi^{(i+1)}=\varphi^{(i)}+f_{\varphi} \tau, \rho^{(i+1)}=\rho^{(i)}+P_{\rho}^{(i+1)} \tau, \\
z^{(i+1)}=z^{(i)}+P_{z}^{(i+1)} \tau, \quad i=1,2, \ldots
\end{gathered}
$$

Подчеркнем, что при вычислении координат используются импульсы, вычисленные в первых операторах. Докажем, что преобразование (13) является каноническим преобразованием. Для этого приведем его к явному виду

$$
\begin{gathered}
P_{\varphi}^{(i+1)}=P_{\varphi}^{(i)}, \quad P_{\rho}^{(i+1)}=P_{\rho}^{(i)}+f_{\rho} \tau, \\
P_{z}^{(i+1)}=P_{z}^{(i)}+f_{z} \tau, \quad \varphi^{(i+1)}=\varphi^{(i)}+f_{\varphi} \tau, \\
\rho^{(i+1)}=\rho^{(i)}+P_{\rho}^{(i)} \tau+f_{\rho} \tau^{2}, z^{(i+1)}=z^{(i)}+P_{z}^{(i)} \tau+f_{z} \tau^{2}, \\
i=1,2, \ldots,
\end{gathered}
$$

и, вычисляя скобку Пуассона (6) полученного преобразования, имеем

$$
\left[P_{\rho}^{(i+1)}, \rho^{(i+1)}\right]=1,\left[P_{z}^{(i+1)}, z^{(i+1)}\right]=1,\left[P_{\varphi}^{(i+1)}, \varphi^{(i+1)}\right]=1 .
$$

Таким образом, алгоритм численного интегрирования уравнений Гамильтона (10) движения нерелятивистской частицы в магнитном поле осуществляет каноническое преобразование фазового пространства и устойчив к накоплению погрешности счета.

Для сравнения рассмотрим преобразование фазового пространства, соответствующее методу Эйлера численного интегрирования

$$
\begin{gathered}
P_{\varphi}^{(i+1)}=P_{0}, P_{\rho}^{(i+1)}=P_{\rho}^{(i)}+f_{\rho} \tau, P_{z}^{(i+1)}=P_{z}^{(i)}+f_{z} \tau, \\
\varphi^{(i+1)}=\varphi^{(i)}+f_{\varphi} \tau, \quad \rho^{(i+1)}=\rho^{(i)}+P_{\rho}^{(i)} \tau, \\
z^{(i+1)}=z^{(i)}+P_{z}^{(i)} \tau, \quad i=1,2, \ldots .
\end{gathered}
$$

Вычисляя скобку Пуассона, для преобразования Эйлера получим

$$
\begin{gathered}
{\left[P_{\rho}^{(i+1)}, \rho^{(i+1)}\right]=-\nabla_{\rho} f_{\rho} \tau^{2} \neq 1,} \\
{\left[P_{z}^{(i+1)}, z^{(i+1)}\right]=-\nabla_{z} f_{z} \tau^{2} \neq 1, \quad\left[P_{\varphi}^{(i+1)}, \varphi^{(i+1)}\right]=1 .}
\end{gathered}
$$

Нарушение условия каноничности ведет к быстрому накоплению погрешности счета в методе Эйлера. Тот же недостаток имеют и все другие известные алгоритмы, например алгоритм Рунге-Кутта [2].

Укажем некоторые свойства алгоритма (13). Поскольку в алгоритме сначала вычисляются импульсы, 
которые затем используются для вычисления координат, в теории канонического интегрирования его принято называть „импульс-координата“. Меняя местами порядок вычисления, можно получить алгоритм „координата-импульс“

$$
\begin{gathered}
\varphi^{(i+1)}=\varphi^{(i)}+f_{\varphi} \tau, \quad \rho^{(i+1)}=\rho^{(i)}+P_{\rho}^{(i+1)} \tau, \\
z^{(i+1)}=z^{(i)}+P_{z}^{(i+1)} \tau, \\
P_{\varphi}^{(i+1)}=P_{\varphi}^{(i)}, \quad P_{\rho}^{(i+1)}=P_{\rho}^{(i)}+f_{\rho}^{(i+1)} \tau, \\
P_{z}^{(i+1)}=P_{z}^{(i)}+f_{z}^{(i+1)} \tau, \quad i=1,2, \ldots,
\end{gathered}
$$

где $f_{\varphi}^{(i+1)}=f_{\varphi}\left(\rho^{(i+1)}, z^{(i)}\right), f_{z}^{(i+1)}=f_{\varphi}\left(\rho^{(i)}, z^{(i+1)}\right)$.

Из эквивалентности импульсов и координат в гамильтоновой механике следует, что алгоритм „координата-импульс“" также будет каноническим.

Как видно из явного выражения (14), алгоритм „импульс-координата“ имеет первый порядок точности при вычислении импульсов и второй - при вычислении координат. Для алгоритма „координата-импульс“ ситуация будет обратной. Таким образом, порядок точности обоих методов равен 1.5. Отсюда следует, что оптимальным будет использование алгоритма „импульс-координата-координата-импульс“. В этом случае порядок точности оказывается близок к двум без увеличения общего количества вычисляемых операторов.

В заключение заметим, что полученные алгоритмы содержат минимально возможное количество операторов. В частности, в сравнении с алгоритмом Эйлера (13) отсутствует оператор, фиксирующий значение старых координат. В этой связи канонические алгоритмы обладают максимально возможным быстродействием.

\section{Релятивистская частица в постоянном магнитном поле}

Пусть по-прежнему векторный потенциал (8) постоянен во времени, но скорость частицы может быть близкой к скорости света. В частности, этот случай реализуется в бета-спектрометрах [5].

Запишем функцию и динамические уравнения Гамильтона (1), (3) в цилиндрической системе координат $O \rho \varphi z$

$$
\begin{gathered}
H=\left[m^{2} c^{4}+c^{2} P_{\rho}^{2}+c^{2} P_{z}^{2}+c^{2} \rho^{2}\left(P_{\varphi}-e A_{\varphi}\right)^{2}\right]^{0.5} \\
\dot{P}_{\rho}=-c^{2} H^{-1} \rho\left(P_{\varphi}-e A_{\varphi}\right)\left(P_{\varphi}-e A_{\varphi}-e \rho \nabla_{\rho} A_{\varphi}\right) \\
\dot{\rho}=c^{2} H^{-1} p_{\rho}, \quad \dot{P}_{\varphi}=0 \\
\dot{\varphi}=c^{2} H^{-1} \rho^{2}\left(P_{\varphi}-e A_{\varphi}\right) \\
\dot{P}_{z}=c^{2} e H^{-1} \rho^{2}\left(P_{\varphi}-e A_{\varphi}\right) \nabla_{\rho} A_{\varphi}, \quad \dot{z}=c^{2} H^{-1} P_{z}
\end{gathered}
$$

где $P_{\rho}, P_{\varphi}, P_{z}$ - компоненты обобщенного импульса в системе $O \rho \varphi z$.
Поскольку рассматриваемая нами система является консервативной, имеет место закон сохранения энергии

$$
\left[m^{2} c^{4}+c^{2} P_{\rho}^{2}+c^{2} P_{z}^{2}+c^{2} \rho^{2}\left(P_{\varphi}-e A_{\varphi}\right)^{2}\right]^{0.5}=H_{0},
$$

где значение функции Гамильтона $H_{0}$ определяется начальными условиями. Поэтому систему (16) можно записать в виде

$$
\begin{gathered}
\dot{P}_{\varphi}=0, \dot{P}_{\rho}=c^{2} H_{0}^{-1} f_{\rho}, \dot{P}_{z}=c^{2} H_{0}^{-1} f_{z}, \dot{\varphi}=c^{2} H_{0}^{-1} f_{\varphi}, \\
\dot{\rho}=c^{2} H_{0}^{-1} P_{\rho}, \quad \dot{z}=c^{2} H_{0}^{-1} P_{z},
\end{gathered}
$$

где мы ввели обозначения

$$
\begin{aligned}
& f_{\rho}=-\rho\left(P_{\varphi}-e A_{\varphi}\right)\left(P_{\varphi}-e A_{\varphi}-e \rho \nabla_{\rho} A_{\varphi}\right), \\
& f_{\varphi}=\rho^{2}\left(P_{\varphi}-e A_{\varphi}\right), \quad f_{z}=e \rho^{2}\left(P_{\varphi}-e A_{\varphi}\right) \nabla_{z} A_{\varphi} .
\end{aligned}
$$

Формально система (21) эквивалентна системе (12), поэтому мы сразу получаем канонические алгоритмы численного интегрирования „импульс-координата“ и „координата-импульс“ для уравнений Гамильтона (19)

$$
\begin{gathered}
P_{\varphi}^{(i+1)}=P_{0}, \quad P_{\rho}^{(i+1)}=P_{\rho}^{(i)}+c^{2} H_{0}^{-1} f_{\rho} \tau, \\
P_{z}^{(i+1)}=P_{z}^{(i)}+c^{2} H_{0}^{-1} f_{z} \tau, \\
\varphi^{(i+1)}=\varphi^{(i)}+c^{2} H_{0}^{-1} f_{\varphi} \tau, \rho^{(i+1)}=\rho^{(i)}+c^{2} H_{0}^{-1} P_{\rho}^{(i+1)} \tau, \\
z^{(i+1)}=z^{(i)}+c^{2} H_{0}^{-1} P_{z}^{(i+1)} \tau, \quad i=1,2, \ldots \quad(23) \\
\varphi^{(i+1)}=\varphi^{(i)}+c^{2} H_{0}^{-1} f_{\varphi} \tau, \rho^{(i+1)}=\rho^{(i)}+c^{2} H_{0}^{-1} P_{\rho}^{(i+1)} \tau, \\
z^{(i+1)}=z^{(i)}+c^{2} H_{0}^{-1} P_{z}^{(i+1)} \tau, \quad P_{\varphi}^{(i+1)}=P_{0}, \\
P_{\rho}^{(i+1)}=P_{\rho}^{(i)}+c^{2} H_{0}^{-1} f_{\rho}^{(i+1)} \tau, \\
P_{z}^{(i+1)}=P_{z}^{(i)}+c^{2} H_{0}^{-1} f_{z}^{(i+1)} \tau, \quad i=1,2, \ldots,
\end{gathered}
$$

где по-прежнему $f_{\varphi}^{(i+1)}=f_{\varphi}\left(\rho^{(i+1)}, z^{(i)}\right), \quad f_{z}^{(i+1)}=$ $=f_{\varphi}\left(\rho^{(i)}, z^{(i+1)}\right)$.

Свойства алгоритмов (13) и (17) переносятся на алгоритмы (23), (24).

\section{Релятивистская частица в переменном магнитном поле}

Пусть векторный потенциал (8) изменяется во времени и скорость частицы вследствие индукционного ускорения может стать как угодно близкой к скорости света, как это имеет место в бетатронах [6].

Функция Гамильтона (1) в цилиндрической системе координат $O \rho \varphi z$ будет иметь форму (18), но векторный потенциал будет, кроме того, явной функцией времени $A_{\varphi}=A_{\varphi}(\rho, z, t)$. Значение функции Гамильтона (20) попрежнему будет определяться начальными значениями координат и импульсов, но сама функция будет изменяться вследствие явной зависимости от времени $A_{\varphi}$

$$
H_{0}(t)=\left[m^{2} c^{4}+c^{2} P_{\rho 0}^{2}+c^{2} P_{z 0}^{2}+c^{2} \rho_{0}^{2}\left(P_{\varphi 0}-e A_{\varphi 0}\right)^{2}\right]^{0.5},
$$

где $P_{\rho 0}, P_{z 0}, H_{0}, \rho_{0}, z_{0}$ определяются начальными условиями, $A_{\varphi 0}=A_{\varphi}\left(\rho_{0}, z_{0}, t\right)$ - заданная функция времени. 
Поскольку (25) не зависит от изменения координат, скобки Пуассона (6), вычисленные для системы (19) при явной зависимости векторного потенциала от времени вновь приводят к выполнению условия каноничности (15).

Таким образом, алгоритмы (23), (24) и их свойства распространяются на случай движения частицы в переменном магнитном поле.

\section{Релятивистский осциллятор}

Для верификации полученных результатов осуществим численное интегрирование уравнений движения релятивистского электрона в постоянном магнитном поле бетатронного типа каноническим методом, методом Эйлера и методом Рунге-Кутта. Пусть в плоскости $z=0$ цилиндрической системы координат $O \rho \varphi z$ определена изолиния векторного потенциала в виде окружности радиуса $\rho_{0}, A\left(\rho_{0}\right)=A_{0}$, в окрестности которой векторный потенциал изменяется по закону

$$
\left(A_{\varphi} r\right)^{2}=A_{0}^{2}\left(1+0.5 r^{2}\right), \quad A_{\rho}=A_{z}=0,
$$

где $r=\rho-\rho_{0}$. Рассмотрим движение релятивистского электрона в поле (26) при начальных условиях $r=0$, $z=0, p_{\varphi}=A_{0}, P_{\rho}=P_{\rho 0} \ll p_{\varphi}, P_{\varphi}=0$. Для упрощения анализа введем безразмерные комбинации

$$
\begin{gathered}
t \leftarrow \frac{t c}{\rho_{0}}, \quad r \leftarrow \frac{r}{\rho_{0}}, \quad a \leftarrow \frac{A_{\varphi}}{A_{0}}, \quad h \leftarrow \frac{H}{c e\left(A_{0} \rho_{0}\right)}, \\
e_{0} \leftarrow \frac{m c}{e\left(A_{0} \rho_{0}\right)}, \quad p_{\varphi} \leftarrow \frac{p_{\varphi}}{e c A_{0}}, \quad p_{\rho} \leftarrow \frac{P_{\rho}}{e c\left(A_{0} \rho_{0}\right)} .
\end{gathered}
$$

Запишем функцию Гамильтона и динамические уравнения электрона

$$
\begin{gathered}
h=\left[e_{0}^{2}+p_{\rho}^{2}+\left(p_{\varphi} r\right)^{2}+\left(1+0.5 r^{2}\right)\right]^{0.5}, \\
\frac{d p_{r}}{d t}=-\frac{1}{2} \frac{r}{h}, \quad \frac{d r}{d t}=\frac{p_{r}}{h}, \quad \frac{d \varphi}{d t}=\frac{1}{h} .
\end{gathered}
$$

Функция Гамильтона является интегралом движения, поэтому динамические уравнения можно переписать в виде

$$
\frac{d \varphi}{d t}=\frac{1}{h_{0}}, \quad \frac{d p_{r}}{d t}=-\frac{1}{2} \frac{r}{h_{0}}, \quad \frac{d r}{d t}=\frac{p_{r}}{h_{0}},
$$

где $h_{0}$ - начальное значение функции Гамильтона.

Мы видим, что движение электрона состоит из равномерного движения по окружности изолинии, задаваемым первым уравнением, и гармонических колебаний перпендикулярно окружности, определяемых двумя последними уравнениями. Для сравнительного анализа алгоритмов будем численно интегрировать уравнения гармонических колебаний (29). Запишем алгоритмы интегрирования.

Канонический алгоритм импульс-координата содержит два оператора

$$
p_{r}=p_{r}-0.5 h_{0}^{-1} r \tau, \quad r=r+p_{r} h_{0}^{-1} p_{r} \tau .
$$

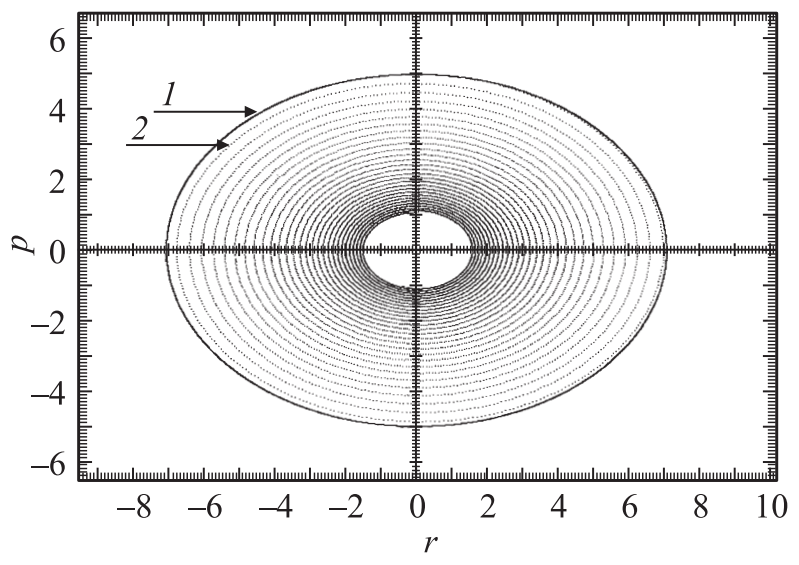

Фазовые траектории релятивистского осциллятора: 1 - интегрирование по каноническому алгоритму, 2 - интегрирование по алгоритму Рунге-Кутта.

Алгоритм Эйлера содержит три оператора

$$
p_{r}^{*}=p_{r}, \quad p_{r}=p_{r}-0.5 h_{0}^{-1} r \tau, \quad r=r+p_{r}^{*} h_{0}^{-1} p_{r} \tau .
$$

Алгоритм Рунге-Кутта 4-го порядка содержит десять операторов

$$
\begin{aligned}
& K_{1}=-0.5 h_{0}^{-1} r \tau, L_{1}=h_{0}^{-1} p_{r} \tau, \\
& K_{2}=-0.5 h_{0}^{-1}\left(r+0.5 L_{1}\right) \tau, L_{2}=h_{0}^{-1}\left(p_{r}+0.5 K_{1}\right) \tau, \\
& K_{3}=-0.5 h_{0}^{-1}\left(r+0.5 L_{2}\right) \tau, L_{3}=h_{0}^{-1}\left(p_{r}+0.5 K_{2}\right) \tau, \\
& K_{4}=-0.5 h_{0}^{-1}\left(r+L_{3}\right) \tau, L_{4}=h_{0}^{-1}\left(p_{r}+K_{3}\right) \tau, \\
& p_{r}=p_{r}+6^{-1}\left(K_{1}+2 K_{2}+2 K_{3}+K_{4}\right), \\
& r=r+6^{-1}\left(L_{1}+2 L_{2}+2 L_{3}+L_{4}\right) .
\end{aligned}
$$

Очевидно, наибольшее быстродействие у канонического алгоритма.

Сравнение устойчивости осуществим, интегрируя систему динамических уравнений релятивистского электрона (28), используя алгоритмы (30) и (32). На рисунке приведены полученные фазовые траектории электрона $p_{r}=p_{r}(r)$ при шаге интегрирования $\tau=0.5$ и числе шагов 10000 . При интегрировании каноническим алгоритмом фазовая траектория представляет собой устойчивый эллипс. Интегрирование методом Рунге-Кутта приводит к фазовой траектории в виде спирали, что означает неустойчивость этого метода к накоплению погрешности счета. Тем более будет неустойчив алгоритм Эйлера, поскольку его можно рассматривать как алгоритм Рунге-Кутта второго порядка.

\section{Заключение}

Мы построили алгоритмы численного интегрирования динамических уравнений для различных случаев движения заряженных частиц в магнитном поле с осевой 
симметрией. Выполнение условия каноничности преобразований фазового пространства, осуществляемого алгоритмами, обеспечивают устойчивость процесса интегрирования к накоплению погрешности счета. Это свойство делает перспективным применение предложенных алгоритмов для расчета технических устройств, использующих движение заряженных частиц в магнитном поле с осевой симметрией.

\section{Список литературы}

[1] Арнольд В.И. Математические методы классической механики. М.: Наука, 1974. 432 с.

[2] Ефимов И.Н., Морозов Е.А. Каноническое интегрирование динамических систем. Екатеринбург. Изд-во Института экономки УрО РАН, 2006. $143 \mathrm{c}$.

[3] Ландау Л.Д. Теория поля. М.: Наука, 1967. С. 460.

[4] Трапезников И.А., Евстафьев А.И., Сапожников В.П., Шабанова И.Н. и др. // ФММ. 1973. Т. 36. № 6. С. 1293 1305.

[5] Зигбан К. Альфа-, бета-, гамма-спектроскопия. М.: Атомиздат, 1969. $567 \mathrm{c}$.

[6] Лебедев А.Н., Шальнов А.В. Основы физики и техники ускорителей. М.: Энергоиздат, 1991. 528 с. 